\title{
Surgeons' Preference for Off-Pump or On-Pump Coronary Artery Bypass Grafting Surgery
}

\author{
GuangPu Fan, PhD, ${ }^{1}$ Xuan Wang, MM, ${ }^{1}$ Chen Chen, MM, ${ }^{1,2}$ Jing Liu, RN, ${ }^{1}$ Yu Chen, MD ${ }^{1}$ \\ ${ }^{1}$ Department of Cardiac Surgery, Peking University People's Hospital, Beijing, China \\ ${ }^{2}$ Cardiac Surgery, China-Japan Union Hospital of Jilin University, Changchun, Jilin, China
}

\section{ABSTRACT}

Background: Surgeon's preference is an important factor in clinical strategy for off-pump (OPCAB) or on-pump (ONCAB) coronary artery bypass graft (CABG) surgery. This study analyzed surgeons' understanding of and propensity for both techniques.

Methods: A survey was performed by self-reported questionnaire. Two sections were included: Q1 questionnaire investigated each surgeon's opinion on the indications of $\mathrm{OPCAB}$ and ONCAB; and Q2 questionnaire investigated each surgeon's choice of OPCAB or ONCAB in different clinical situations.

Results: The questionnaires were sent to 169 surgeons. In Q1, 71.2\% of surgeons indicated that the degree of overlap between the indications of $\mathrm{OPCAB}$ and $\mathrm{ONCAB}$ is $>70 \%$; $55.1 \%$ believed that OPCAB had a wider scope of indications than $\mathrm{ONCAB}$, and $35.3 \%$ believed that ONCAB had a wider scope of indications than OPCAB. In Q2, >70\% of surgeons who responded chose OPCAB for patients with the following characteristics: high risk of stroke, renal dysfunction, pulmonary dysfunction, malignancy, clotting and coagulation disorders, or age $\geq 80$ years. More than $57.5 \%$ of surgeons chose ONCAB for patients with poor target vessels or ventricular enlargement and dysfunction. For novice surgeons, $87.5 \%$ of surgeons chose ONCAB.

Conclusion: Most surgeons surveyed agreed that $\mathrm{OPCAB}$ and $\mathrm{ONCAB}$ are suitable for most patients; however, surgeons' preference for ONCAB or OPCAB varied. Surgeons are more willing to choose ONCAB in the presence of complicated heart conditions and $\mathrm{OPCAB}$ in the presence of serious concomitant diseases.

\section{INTRODUCTION}

In spite of the coexistence of off-pump (OPCAB) and onpump (ONCAB) coronary artery bypass surgery for many years, current evidence is inconclusive regarding their pros

Received Februray 26, 2021; received in revised form March 13, 2021; accepted March 15, 2021.

Correspondence: Yu Chen, MD, Department of Cardiac Surgery, Peking University People's Hospital, No. 11 Xizhimen South Street, 100044, Beijing, China; 010-88324550 (e-mail: micsc@sina.com). and cons. Some well-designed trials, such as ROOBY and CORONARY, reported that OPCAB had no clinical advantages over $\mathrm{ONCAB}$ and even increased the risk of major adverse cardiovascular events or repeat revascularization [Lamy 2016; Shroyer 2017; Hu 20210]. Correspondingly, several large propensity-matched retrospective series and meta-analyses from centers proficient in OPCAB provided evidence in favor of this technique in decreasing risk of death, stroke, acute renal failure, mortality, morbidity, and postoperative length of stay compared with ONCAB [Polomsky 2013; Kowalewski 2016]. Thus, how to make a reasonable choice is a constant clinical question.

Patients routinely accept the surgeon's preferred technique for performing coronary artery surgery [Nishith 2011]. Therefore, a surgeon has to choose OPCAB or ONCAB for individual patients based on the surgical strategy. The choice depends on the patient's coronary artery lesions and comorbidities, the team's ability, some social and economic situations, and most important, the surgeon's training and proficiency in the 2 techniques. This strategy, even for a specific patient, varies by surgeon, institution, and region.

Previous studies mostly focused on skills and outcomes in $\mathrm{OPCAB}$ and $\mathrm{ONCAB}$, rather than surgeon preference. Thus, we surveyed the subjective intention of cardiac surgeons about the indications for OPCAB and ONCAB, as well as their preferences for each technique in different clinical settings.

\section{MATERIALS AND METHODS}

\section{Study Population}

We undertook a cross-sectional, self-reported questionnaire survey of 169 registered cardiac surgeons from China.

\section{Survey Instrument}

The questionnaire included 2 parts: Q1 and Q2. Q1 aimed to investigate the surgeon's opinion about the indications for $\mathrm{OPCAB}$ and $\mathrm{ONCAB}$ (Figure 1). Q2 aimed to investigate the surgeon's choice of OPCAB or ONCAB in different clinical situations (Figure 2).

\section{Administration of the Survey}

Each of the surgeons surveyed was contacted individually by an investigator, to ensure that each surgeon completed the survey only once. Other than the content of the questionnaire itself, the investigator did not undertake any additional explanation. 
There were 2 types of surveys: for on-site investigation, questionnaires were filled out and collected on the spot through academic meetings or hospital visits; and for mobile surveys, the investigator contacted the surgeon through instant messaging software, directly issued questionnaires, and collected the answers.

\section{Statistical Analysis}

Excel 2010 and SPSS 18.0 statistical software were used for data collation and statistical processing. The composition ratio was used as the main statistical index for descriptive analysis, and $\chi^{2}$ test was used for comparison between groups. $P<.05$ was considered statistically significant.

\section{RESULTS}

\section{Results of $Q 1$}

A total of 156 valid Q1 questionnaires were collected. Four groups were defined according to the number of independent surgeries performed: 69 surgeons in group I had not

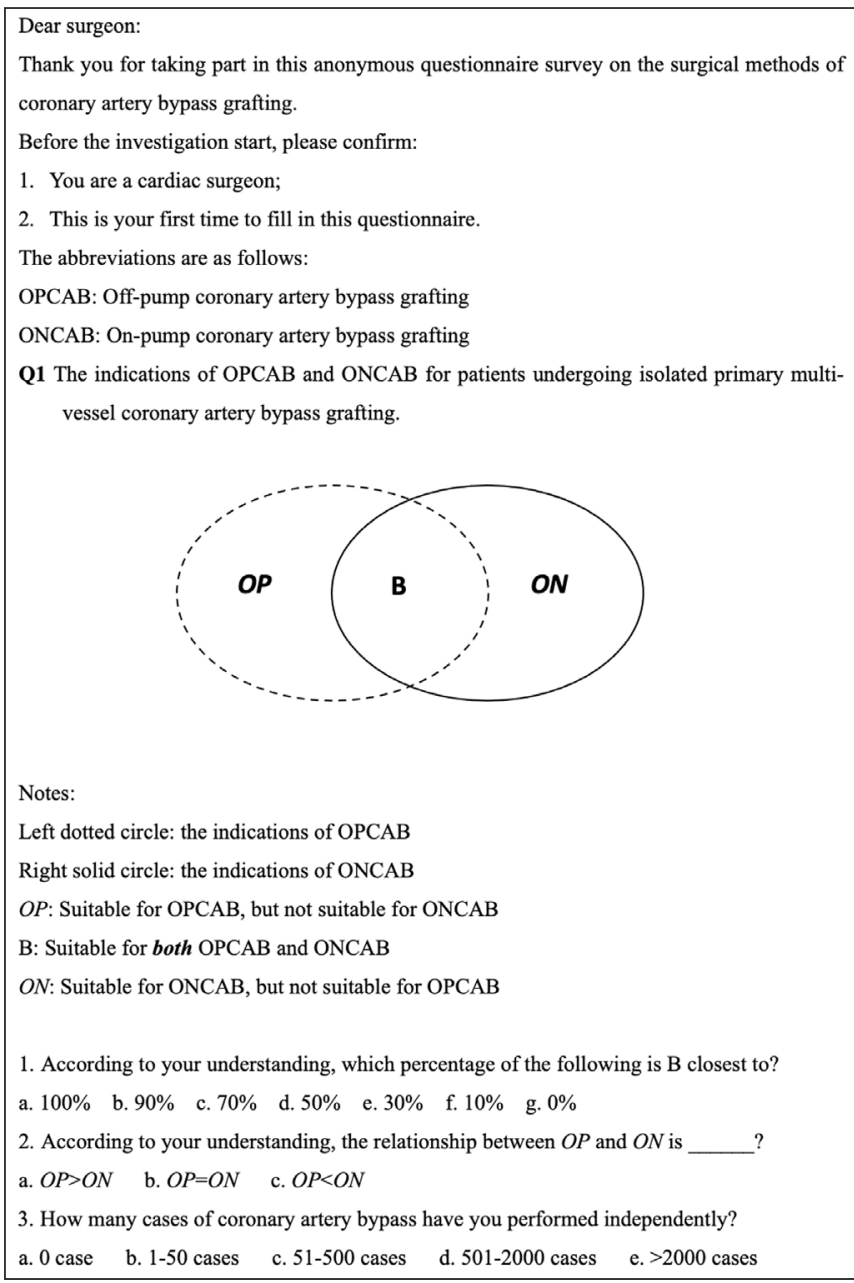

Figure 1. The Q1 questionnaire. yet performed the surgery independently, 26 in group II had performed 1 to 50 independent surgeries, 42 in group III had performed 51 to 2000, and 19 in group IV had performed $>2000$.

\section{Results on the Degree of Overlap between ONCAB} and OPCAB Indications

The $\mathrm{B}$ value, defined as the degree of overlap between the indications for the 2 surgical methods, represents the proportion of patients who are suitable for both $\mathrm{ONCAB}$ and $\mathrm{OPCAB}$. Most surgeons (97.4\%; 152 of 156) did not choose the option

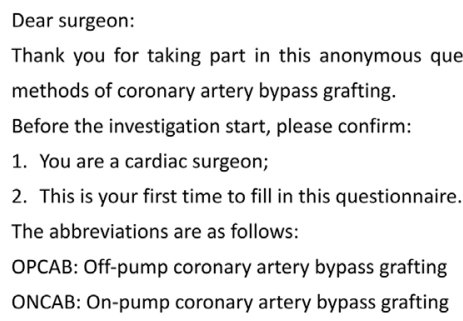

Q2: Under the following circumstances, which technique do you prefer to choose?

A. OPCAB B. ONCAB C. no difference between the two

1. Patients with mild or moderate renal insufficiency

2. Dialysis-dependent patients

3. Patients at high risk of stroke

4. Patients with chronic obstructive pulmonary disease

5. Patients with cardiomegaly and ejection fraction $<40 \%$

6. Multiple diffuse lesions or relatively small coronary vessels

7. Severe left main lesion

8. When an endarterectomy is required

9. Multiple total arterial revascularization

10. intra-myocardial vessles

11. octogenarian patients

12. Very obese patients

13. Patients combined with malignancy

14. Patients with clotting and coagulation disorders

15. Blood shortage or rejection of allogeneic transfusion

16. Novice surgeons in coronary surgery

$\begin{array}{lll}\text { A } & \text { B } & \text { C } \\ \square & \square & \square \\ \square & \square & \square \\ \square & \square & \square \\ \square & \square & \square \\ \square & \square & \square \\ \square & \square & \square \\ \square & \square & \square \\ \square & \square & \square \\ \square & \square & \square \\ \square & \square & \square \\ \square & \square & \square \\ \square & \square & \square \\ \square & \square & \square \\ \square & \square & \square \\ \square & \square & \square \\ \square & \square & \square\end{array}$

Figure 2. The Q2 questionnaire.

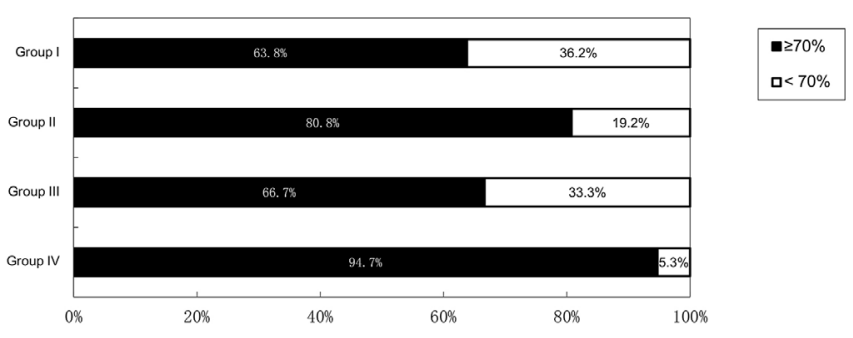

Figure 3. The $B$ value between groups in $Q 1$. B value represents the proportion of patients who are suitable for both $O P C A B$ and $O N C A B$. 
of $100 \%$ for the $\mathrm{B}$ value, thus believing that the indications for $\mathrm{OPCAB}$ and ONCAB did not fully overlap. Those who chose a $\mathrm{B}$ value $\geq 70 \%$ accounted for the majority of all surgeons (71.2\%), and each group showed a similar trend (Figure 3).

Among groups, there were significant differences between surgeons with different volumes of surgery experience $(P=$ $.036)$. In group IV, more surgeons $(94.7 \%)$ chose a B value $\geq 70 \%$ (Table 1 ), and the $\chi^{2}$ test of Mantel-Haenszel showed a trend of linear correlation between an increase in operation volume and a larger $\mathrm{B}$ value $(P=.050)$.

Results on the Scope of ONCAB and OPCAB Indications

Regarding the scope of $\mathrm{OP}$ and $\mathrm{ON}$ indications, $\mathrm{OP}>$ ON means OPCAB has a wider scope of indications than $\mathrm{ONCAB}, \mathrm{OP}<\mathrm{ON}$ means $\mathrm{ONCAB}$ has a wider scope of indications than $\mathrm{OPCAB}$, and $\mathrm{OP}=\mathrm{ON}$ means the same scope of indications for both. In response to this survey question, more surgeons $(55.1 \%)$ chose $\mathrm{OP}>\mathrm{ON}$, and thus believed that OPCAB had a larger suitable population of patients. This tendency could be found in each group and did not differ significantly between groups $(P=.818)$ (Table 2; Figure 4).

\section{Results of $Q 2$}

A total of 120 valid Q2 questionnaires were collected. More than $70 \%$ of surgeons surveyed were inclined to choose

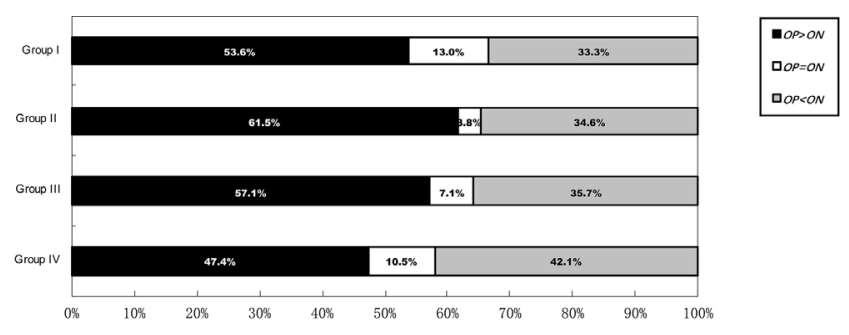

Figure 4. The relationship between $\mathrm{OP}$ value and $\mathrm{ON}$ value in $\mathrm{Q} 1 . \mathrm{OP}$ and $O N$ represent the scope of OPCAB and ONCAB indications, respectively.

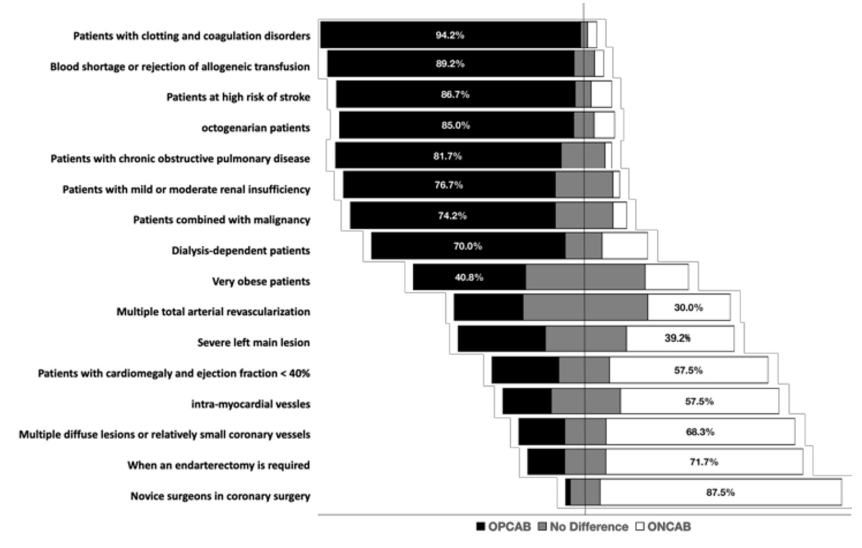

Figure 5. Surgeon preferences for $O P C A B$ and $O N C A B$ in different clinical conditions.
OPCAB when patients had more concurrent diseases such as high risk of stroke, renal dysfunction, pulmonary dysfunction, malignancy, and clotting and coagulation disorders or were octogenarians. More than $57.5 \%$ of surgeons surveyed were inclined to choose ONCAB when the difficulties and challenges of surgery were focused on the heart itself, such as poor target vessels and ventricular enlargement and dysfunction. One of the noteworthy results was that for novice surgeons, the choice of ONCAB achieved a very high consensus (87.5\%) (Figure 5).

\section{DISCUSSION}

Coronary surgery has a history of $>60$ years, during which ONCAB has been the dominant approach. For $>20$ years, the OPCAB approach has aimed to improve the outcome of coronary artery bypass surgery by reducing the damage caused by extracorporeal circulation. However, there has been insufficient evidence to prove the relative merits of either approach [Lamy 2016]. Whether OPCAB and ONCAB are applicable to the same or different types of patients remains to be further explored.

In this study, most surgeons agreed that indications for the 2 techniques partially overlap (B value), but are not completely superimposable (OP value and $\mathrm{ON}$ value), which suggests that neither of the techniques can handle all practical clinical situations. This is consistent with some of the cases that we envision encountering, such as severe aortic calcification or hemodynamic instability. In our survey, most surgeons believed that OPCAB and ONCAB have different scopes of indications, and that OPCAB expands the indications of traditional onpump technique, but there are still a need for ONCAB that OPCAB cannot meet. Accordingly, surgeons should consider more of the factors (OP or ON values) that cannot be fully covered by a technique and comprehend the limitations of one technique and the advantages of the other. Being aware of this may help surgeons to make rational strategic choices.

The $\mathrm{B}$ value of $\mathrm{Q} 1$ represents the overlap range of $\mathrm{OPCAB}$ and ONCAB indications, which may largely depend on the

Table 1. The B Value between Groups in Q1*

\begin{tabular}{lcccc}
\hline Group & $\mathrm{n}$ & $\geq 70 \%$ & $<70 \%$ & $P$ Value \\
\hline I & 69 & $44(63.8)$ & $25(36.2)$ & \\
II & 26 & $21(80.8)$ & $5(19.2)$ & \\
III & 42 & $28(66.7)$ & $14(33.3)$ & \\
IV & 19 & $18(94.7)$ & $1(5.3)$ & \\
Total & 156 & $111(71.2)$ & $45(28.8)$ & .036 \\
\hline
\end{tabular}

*Data are n (\%) unless noted otherwise. Group I, surgeons without independent surgical experience; group II, surgeons with 1 to 50 cases; group III, surgeons with 51 to 2000 cases; and group IV, surgeons with $>2000$ cases. $B$ value represents the proportion of patients who are suitable for both OPCAB and ONCAB. 
surgeon's training experience and surgical ability. Many studies have proposed that OPCAB training and operation volume have a great influence on the clinical outcomes of OPCAB [Taggart 2020]. Surgeons who have received more OPCAB training and performed more OPCAB operations may be more inclined to choose $\mathrm{OPCAB}$ as the surgical strategy for a new patient. For surgeons with less OPCAB training and less surgery experience, ONCAB is obviously a favorite option. However, it should be emphasized that this only an explanation of the differences between the techniques, not a direct reflection of the effectiveness of treatment. In the questionnaire results, we also noticed a trend that surgeons with $>2000$ surgeries chose a larger range of $\mathrm{B}$ values, whereas those without independent surgery chose a relatively small range of $\mathrm{B}$ values. More specific evidence is that the ONCAB option in Q2 for novice surgeons accounted for $87.5 \%$, reaching first place among all reasons for ONCAB selection.

Although surgeons subjectively believe that OPCAB or ONCAB can be applied to most patients, the choice between $\mathrm{OPCAB}$ and $\mathrm{ONCAB}$ may be controversial for patients in special subgroups or under special clinical conditions. Q2 was designed to investigate these possibly controversial issues. The descriptive results reflect the tendency of surgeons to opt for OPCAB surgery in patients with severe comorbidities and complex clinical conditions and for ONCAB surgery in patients with complex cardiac conditions. However, the consistency between subjective knowledge and objective evidence is also worth discussing. For example, the surgeons in this study were subjectively inclined to choose OPCAB surgery for elderly patients ( $\geq 80$ years), whereas the randomized results of the GOPCABE trial showed no difference between $\mathrm{OPCAB}$ and $\mathrm{ONCAB}$ surgery in 5-year survival rates, myocardial infarction, or revascularization in elderly patients [Diegeler 2019].

Another controversial example is the case of severe left main lesion, in which surgeons' opinions were scattered, and the selection of all options were almost equal in number. The EXCEL study, in contrast, clearly stated that OPCAB increased 3-year all-cause mortality in patients with left main lesion [Benedetto 2019]. Other controversial questions in Q2 may also be points of interest and need more objective evidence to confirm.
Experts admit that the choice between OPCAB and $\mathrm{ONCAB}$ is an individualized experience, a combination of a surgeon's technical proficiency and outcome cognition [Puskas 2019]. The results of this study-including the range of $\mathrm{B}$ values, the relationship between $\mathrm{OP}$ and $\mathrm{ON}$, and the choice of doctors in different clinical situations-fully illustrate the enthusiasm of the surveyed doctors for OPCAB surgery. If a technique is considered to be difficult and challenging, surgeons would be motivated to pursue it if its outcome is not inferior. In other words, OPCAB, which is considered to be more technically difficult, will still be popular with surgeons, and the current evidence does not clearly suggest its superiority over ONCAB.

The limitations of this study are mainly in 2 aspects. First, the surgeons surveyed are only from China. It is known that the ratio of OPCAB to ONCAB in Asian countries is significantly higher than in Europe and the United States, which may make the survey results biased to OPCAB. Second, some misunderstanding of the topics or questions is possible, leaving a small number of surgeons surveyed struggling with options such as "I can do" versus "I should do," and balancing surgical ability and clinical outcomes.

\section{CONCLUSIONS}

Most surgeons surveyed in this study agreed that OPCAB and $\mathrm{ONCAB}$ are suitable for most patients. However, the overlap of the indications between OPCAB and ONCAB varied among surgeons with different volumes of surgical experience. More surgeons with a large volume of surgery ( $>2000$ cases) recognized that the overlap is relatively large. As for the scope of indications for the 2 operations, more surgeons believed that $\mathrm{OPCAB}$ indications are more extensive. On a subjective level, surgeons are more willing to choose $\mathrm{ONCAB}$ when there are adverse factors related to the heart itself, such as poor target vessels or ventricular enlargement and dysfunction. OPCAB is preferred by surgeons in the presence of more serious concomitant factors, such as advanced age, high risk of stroke, renal dysfunction, pulmonary dysfunction, malignancy, and clotting and coagulation disorders. For beginners in coronary surgery, ONCAB is preferred.

Table 2. The Relationship between OP Value and ON Value in Q1*

\begin{tabular}{|c|c|c|c|c|c|}
\hline I & 69 & $37(53.6)$ & $9(13.0)$ & $23(33.3)$ & \\
\hline III & 42 & $24(57.1)$ & $3(7.1)$ & 15 (35.7) & \\
\hline IV & 19 & $9(47.4)$ & $2(10.5)$ & $8(42.1)$ & \\
\hline
\end{tabular}

*Data are n (\%) unless noted otherwise. Group I, surgeons without independent surgical experience; group II, surgeons with 1 to 50 cases; group III, surgeons with 51 to 2000 cases; and group IV, surgeons with >2000 cases. OP and ON represent the scope of OPCAB and ONCAB indications, respectively. 


\section{REFERENCES}

Benedetto U, Puskas J, Kappetein AP, et al. Off-pump versus on-pump bypass surgery for left main coronary artery disease. J Am Coll Cardiol 2019;74:729-740.

Diegeler A, Börgermann J, Kappert U, et al. Five-year outcome after off-pump or on-pump coronary artery bypass grafting in elderly patients. Circulation 2019;139:1865-1871.

Hu S, Zheng Z, Yuan X, et al. Increasing long-term major vascular events and resource consumption in patients receiving off-pump coronary artery bypass: A single-center prospective observational study. Circulation 2010;121:1800-1808.

Kowalewski M, Pawliszak W, Malvindi PG, et al. Off-pump coronary artery bypass grafting improves short-term outcomes in high-risk patients compared with on-pump coronary artery bypass grafting: Metaanalysis. J Thorac Cardiovasc Surg 2016;151:60-77.

Lamy A, Devereaux PJ, Prabhakaran D, et al. Five-year outcomes after off-pump or on-pump coronary-artery bypass grafting. N Engl J Med 2016;375:2359-2368.
Nishith N. Patel, Enoch Akowuah, Jonathan A, et al. Patient preference for coronary artery bypass graft surgery performed on the arrested or beating heart: A questionnaire study. Eur J Cardiothorac Surg 2011;40:1282-1284.

Polomsky M, He X, O’Brien SM, et al. Outcomes of off-pump versus on-pump coronary artery bypass grafting: Impact of preoperative risk. J Thorac Cardiovasc Surg 2013;145:1193-1198.

Puskas JD, Gaudino M, Taggart DP. Experience is crucial in off-pump coronary artery bypass grafting. Circulation 2019;139:1872-1875.

Shroyer AL, Hattler B, Wagner TH, et al. Five-year outcomes after on-pump and off-pump coronary-artery bypass. N Engl J Med 2017;377:623-632.

Taggart DP, Gaudino MF, Gerry S, et al. Arterial Revascularization Trial Investigators. Ten-year outcomes after off-pump versus on-pump coronary artery bypass grafting: Insights from the Arterial Revascularization Trial. J Thorac Cardiovasc Surg 2020;S0022-522330427-X. 